\title{
A standard-based Body Sensor Network system proposal
}

\author{
Enrique Dorronzoro, Ana Verónica Medina, Isabel Gómez, José Antonio \\ Gómez, and Manuel Merino Monge \\ Departamento de Tecnología Electrónica, Universidad de Sevilla, \\ Escuela Técnica Superior de Ingeniería Informática, Spain \\ enriquedz@dte.us.es, \{vmedina, igomez, manmermon, jgomezdte\}@.us.es
}

\begin{abstract}
Body Area Networks are a solution to remote monitoring in order to acquire vital signals of patients. Actual sensors provide its own interface which makes more difficult to integrate them in a system. Using standardized protocols and interfaces increases the usability and the integration of different sensors, to achieve this goal the IEEE 1451 standard has been defined. This paper presents a proposal of a telemedicine system, with an open implementation of the IEEE 1451 standard, to be used in several different situations.
\end{abstract}

Key words: IEEE 1451, telemedicine, BSN, ZigBee, BitCloud Stack

\section{Introduction}

Telemedicine systems provide remote diagnosis and treatment. Actual approaches to the telemedicine focus on:

- Reinforce the therapy and medication plans monitoring.

- Telepresence systems.

- Localization information.

- Vital sign acquisition.

- Patient monitoring, inside and outside the hospital.

Even there are many solutions and proposals the main problem it is found when the solutions presented are heterogeneous. Not following a standard implies issues in order to be integrated in existing or new medical systems. Actual systems present a solution to concrete problems and they are not flexible enough to adapt to new requirements.

One of the previously mentioned objectives of the telemedicine systems is the remote patient monitoring. The main idea is to control the vital sings of the patient when he is moving inside or outside the hospital. At this environment, Body Sensor Network $\overline{\text { BSN }}$ s are a solution in order to acquire those vital signs from the patients. These networks can obtain remotely data about sensors that measure different parameters. They are composed by several nodes that send the data to a central device, a device in charge of process it and store it. 
BSN allow the mobility of the patient while vital signs are being acquired, blood pressure, ECG, [13, pulse, temperature...

When dealing with the medical data, there are several standards to struct the information that it is sent from several devices. Main existing standards are:

- DICOM (Digital Imaging and Communications in Medicine) [6], defines a standard for medical images.

- HL7 (Health Level 7) 7] , defines the exchange between different medical information systems. Specifies a simple data structure composed by segments, data types and labeled fields.

- EN13606, describes the information required for communications between systems and services that provide or require clinic data.

- X73, ISO/IEEE11073[12], is a family of standards to provide total connectivity between medical devices and computers.

Even these are implemented standards for medical applications these standards are not adequate when implementing sensor networks. Commercial solutions use its own implementation(physical interface and protocol) when communicating with medical devices.

IEEE 1451[15] standard is designed to eliminate this vendor implementations and provide a common interfaces to sensors and actuators (transducers). This standard will provide Plug and Play and flexibility to connect different kinds of sensors to a network, providing the possibility of using different types of networks topologies.

At the following section it is an overview of the proposed system, at section 3 there is a description the BSN, including an IEEE 1451 standard and ZigBee overview and the system design proposal. Finally, conclusions are presented at section 4

\section{System overview}

This paper presents a proposal of a telemonitoring system with an open implementation of the IEEE 1451 standard. The system is designed to be flexible and it can be easily adapted to different kinds of environments. This system versatility will allow its adaptation to be used in various situations like remote patient monitoring inside and outside the hospital, catastrophe response, rehabilitation. The telemedicine system follows the structure presented in the fig 1 .

The tele-monitoring system is composed by two main blocks, a sensor network and a personal server. The personal server acquires the data provided by the sensor network, Body Area Network, and transmit this data along a WAN(Wide Area Network). Communication along the WAN is supported by different transmission technologies depending on the scenario(home, rescue, hospitals,etc).

Using the previously shown structure it is common to find several different sensors that measure, bloody pressure 9], skin temperature, ECG[5], EEG[4], etc. As previously mentioned, the data acquired by the sensors is transmitted 


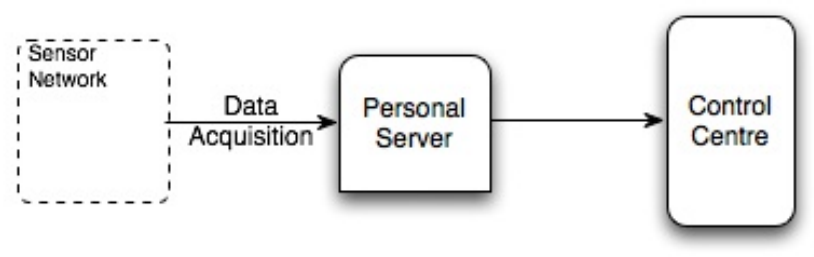

Fig. 1. Personal server acquires the data from the sensor network and it is transmitted to a control centre.

to the personal server using different technologies 14, wireless LAN, BLuetooth, ZigBee, RF, etc.

As most of the available sensor are wired in order to provide them of wireless capabilities many of the recent publications use motes. The most used motes are Mica2 and Micaz, by Crossbow, and propose them to acquire biological [4] and environment 11] signals. Because of the lower power consumption [16] ZigBee is the chosen technology to be implemented in these motes. Sleeping mode of the remote motes allow the system to save more energy than using other technologies, there are also some publications with proposals to save more power using Zigbee 8]. ZigBee can also provide localization with an error rate of two meters [10. The main problem when dealing with ZigBee is the interferences at its operate at the $2 \mathrm{GHz}$ band as Wifi and Bluetooth.

Because of the mote limitations it is common not to make any data processing before sending it. Even there are some publications 4 about making some kind of preprocessing in order to reduce the amount of data to be sent but it is not always possible.

The personal server sent the acquired data to de control center using wide transmission technologies as 3G, GPRS or GSM. There are other alternatives as using the Short Message Service provided by the cellphone providers[8] even it is possible to send data by this method but the amount of data that can be sent it is not appropriated for some applications.

Some papers present solutions to these systems [2 9, 9 1] transmitting vital signs as ECG, pulse, etc. But these papers use vendor interfaces and protocols when connecting the sensors to a personal server device. The solutions are restricted to the presented sensors not allowing different manufacture's sensors.

\section{Body Area Network}

At this section it is described the IEEE 1451 standard and ZigBee standard and the way they are applied to the presented proposal. 


\subsection{IEEE 1451 standard}

This standard defines the connection between sensors. In order to provide sensors with a common interface and provide them of network capabilities there have been defined:

The Transducer Interface Module (TIM) module contains the interface to the transducer. It is in charge of signal conditioning, convert the signal form analog to digital or digital to analog, and in many cases it contains the transducer(one or more).

The Network Capable Application Processor NCAP module implements the hardware and software to provide network services to the TIM.

It also provides the TEDS (Transducer Electronic Data sheet) that contains the required information to identify, characterize, calibrate and use the data provided by an analog or digital sensor.

By this structure Fig. 2 a sensor has a standardized interface and his behavior is specified by its TEDS so it can be added to a system as a plug and play device.
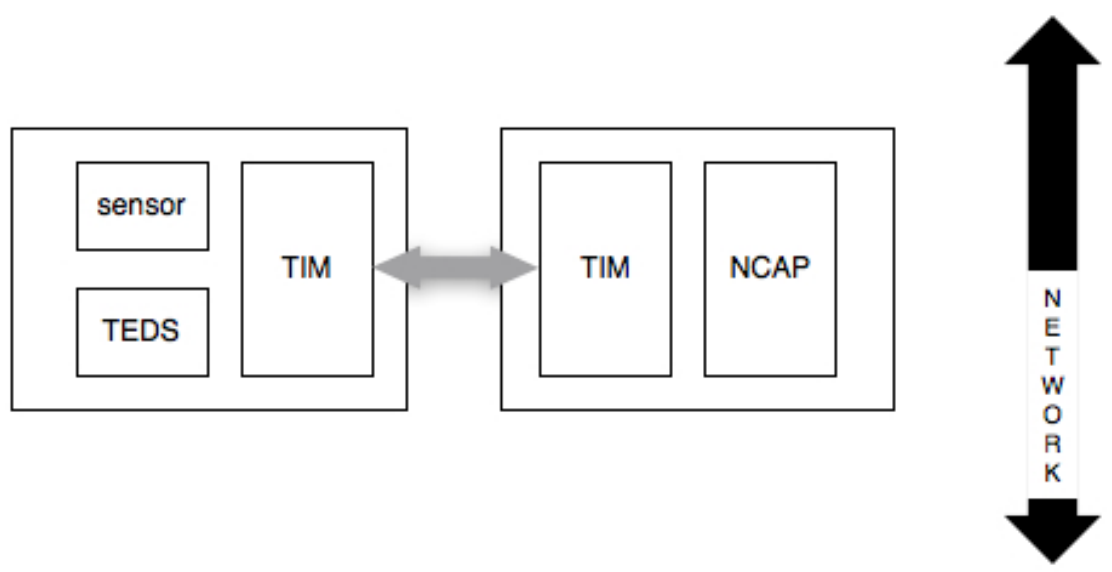

Fig. 2. 1451 sensor system structure.

IEEE standard is divided in six subsections that describe the interface for different kinds of networks connections:

- 1451.0 defines the functions, commands and TEDS for a physical level.

- 1451.1 defines the specification of a common interface for a smart transducer.

- 1451.2 defines the interface between the NCAP and the TEDS for point-topoint connections.

- 1451.3 defines the interface between the NCAP and the TEDS for multi-drop connections.

- 1451.4 defines an interface for analogical transducers and digital operational modes. 
- 1451.5 defines the interface between the NCAP and the TEDS for wireless transducers.

- 1451.6 defines the interface between the NCAP and the TEDS for CANopen networks.

- 1451.7 defines the interface and protocol between transducers and RFID systems.

It is structured as it is represented in fig. 3 .

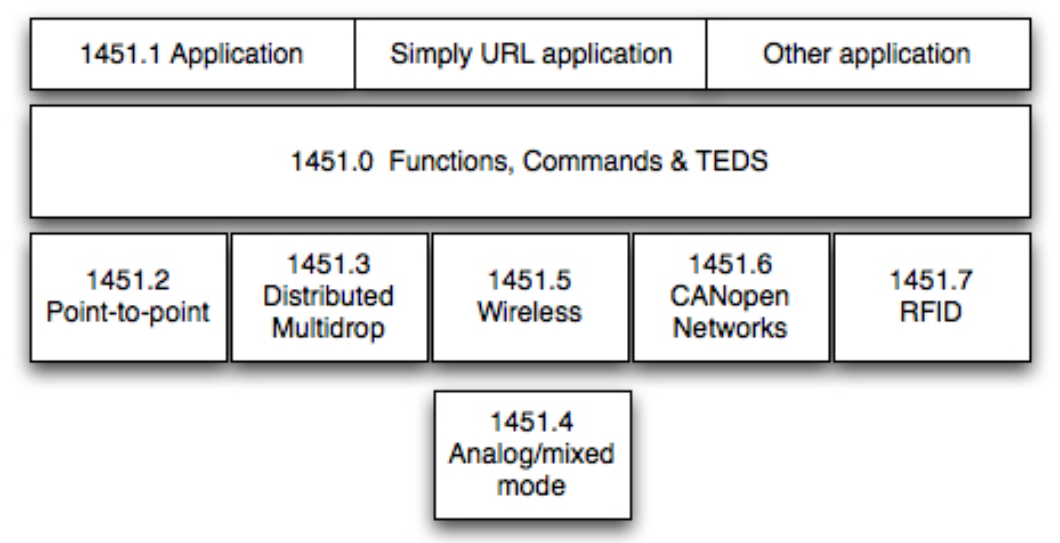

Fig. 3. Layer structure of the IEEE 1451 standard.

\subsection{ZigBee}

IEEE 802.15 .4 is a standard which specifies the physical layer and media access control for low-rate wireless personal area networks (LR-WPANs). It offers short range and low bandwidth but with the benefit of low power consumption. It is mainly use at industrial control, embedded sensors, and it is also adequate for healthcare systems. Healthcare systems can benefit from this technology as a node can go to sleep and wake up when a new task is coming, saving battery with this behavior.

ZigBee is an implementation based in this standard. It provides low power and low duty-cycle sensors. A node can connect to a network in about $30 \mathrm{~ms}$ and when a node is at sleep state it can wakes up in about $15 \mathrm{~ms}$.

ZigBee uses ISM frequency band, mainly at the $2.4 \mathrm{GHz}$ Band. And the cost of the hardware is cheaper than Bluetooth.

Compared with the OSI model ZigBee Alliance only specifies four layers, Physical, Data Link, Network and Application.

There are three different kinds of ZigBee devices regarding its role at the network: 
- ZigBee Coordinator ( $\mathbf{Z C})$ : There must be one coordinator per network. They are in charge of controlling the network and creating the the PAN (Personal Area Network).

- ZigBee Router (ZR): It is charge of routing data in order to make the PAN bigger.

- ZigBee End Device (ZED): End devices transmit information to ZR or $\mathrm{ZC}$ devices, they cannot route data.

Based at functionality another classification is presented:

- Full Function Device (FFD): Devices with network router function, this devices role can be $\mathrm{ZC}$ or $\mathrm{ZR}$

- Reduced Function Device (RFD): Easy and cheap to implement, can enter in a sleep state to minimize the power consumption. The role of this device must be ZED

ZigBee topologies are presented at fig. 4. There are three different topologies:

- Star topology. The communication is established between a central device, a (ZC), and the other ZigBee devices, powered by batteries. This central device is called PAN coordinator and it is unique at the network. It shall be wired powered.

- Mesh topology. It is similar to the star topology. The difference between them is that any device can connect with other device, not just the PAN coordinator.

- Cluster tree topology. Cluster tree is a special scenario for a mesh topology where most of the devices are FFD and RFD are connected to the network as leaves at the end of each branch. One of the FFD is the PAN coordinator and the others can provide synchronization and coordination services.

\subsection{BSN design}

The BSN scheme is presented in fig. 5

The different kind of sensors are connected to motes that send the information required to the personal server.

The proposed mote is a MeshBean by Meshnetic. These motes are based on ZigBit, ZigBit Amp and ZigBit 900 RF module with a matched antenna.ZigBit

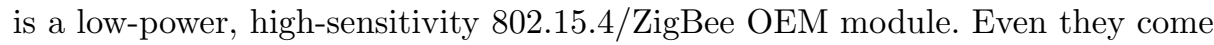
with two built in sensors(temperature and light), it also support extension connector allowing easy access to the modules interfaces.

To perform the communication between these networks elements it is proposed to use Bitcloud. BitCloud is a full-featured embedded software stack from Atmel. The stack provides a software development platform for reliable, scalable, and secure wireless applications running on Atmel wireless platforms.

Common medical sensors are wired sensors. Because of this, sensors are connected to the previously described motes to provide them of network capabilities. The connection between these two devices is done using the Analog/Digital 

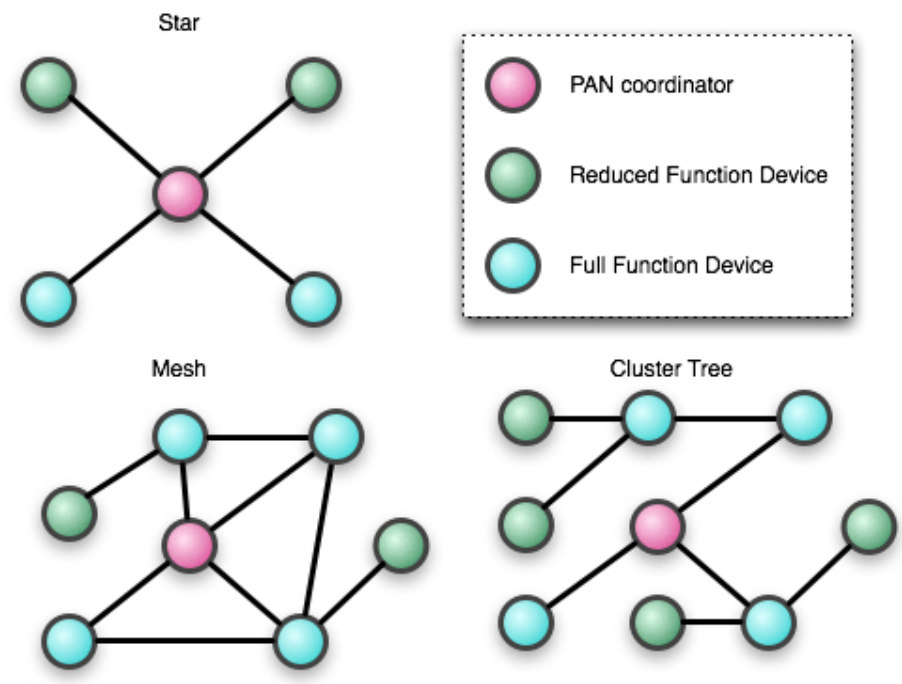

Fig. 4. Zigbee topologies.

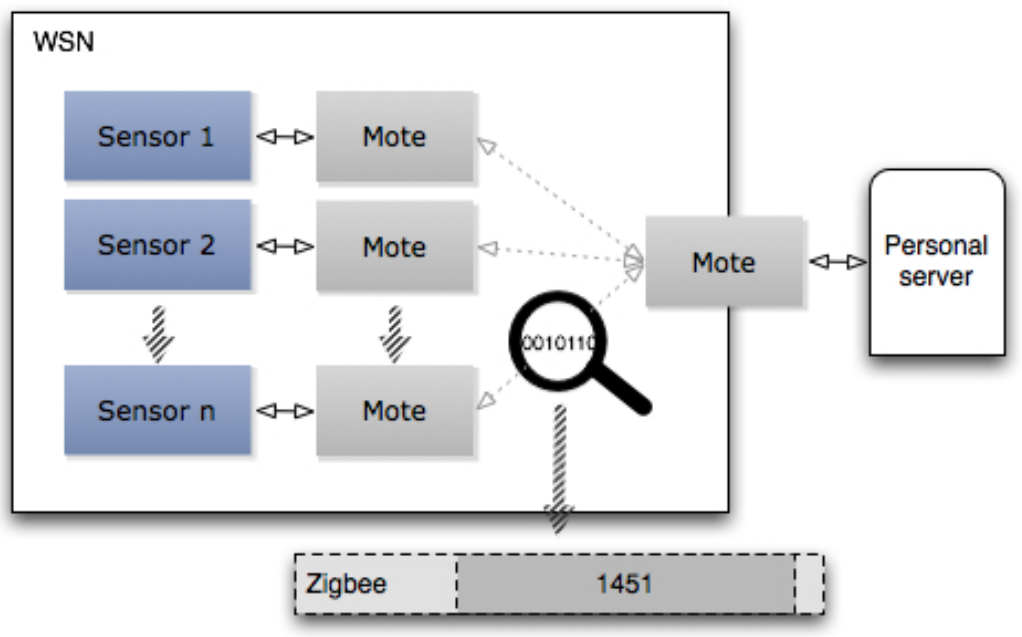

Fig. 5. Wireless sensor network. 
converter included at the mote. When using a Meshnetics mote this physical connection is done connecting the sensor to the pins 15 and $16 / 17 / 18$ as it is specified in the mote data sheet(fig 6) of the expansion slot(fig 7). Providing $8 / 10$ bits of resolution with a reference level of $1.250 \mathrm{~V}$.

\begin{tabular}{|c|c|c|c|}
\hline Pin & Name & 1/O & Description \\
\hline 14 & USART_CLK & Input & $\begin{array}{l}\text { This is Clock Data Pin for USARTO interface } \\
\text { of the ZigBit module. It is connected directly to } \\
\text { the USARTO_EXTCLK pin of the module. Digital } \\
\text { logic level. For details, refer to ZigBit datasheet } \\
\text { [1]. }\end{array}$ \\
\hline 15 & GND & & Digitalanalog ground \\
\hline 16 & ADC_INPUTI & Input & $\begin{array}{l}\text { ADC input. This pin is connected directly to the } \\
\text { ADC INPUT } 1 \text { pin of the module. For details, } \\
\text { refer to ZigBit datasheet [1]. }\end{array}$ \\
\hline 17 & ADC_INPUT2 & Input & $\begin{array}{l}\text { ADC input. This pin is connected directly to the } \\
\text { ADC_INPUT } 2 \text { pin of the module. For details, } \\
\text { refer to ZigBit datasheet [1]. }\end{array}$ \\
\hline 18 & ADC_INPUT3 & Input & $\begin{array}{l}\text { ADC input. This pin is connected directly to the } \\
\text { ADC INPUT } 3 \text { pin of the module. For details, } \\
\text { refer to ZigBit datasheet [1]. }\end{array}$ \\
\hline
\end{tabular}

Fig. 6. Meshnetic mote Pin mapping

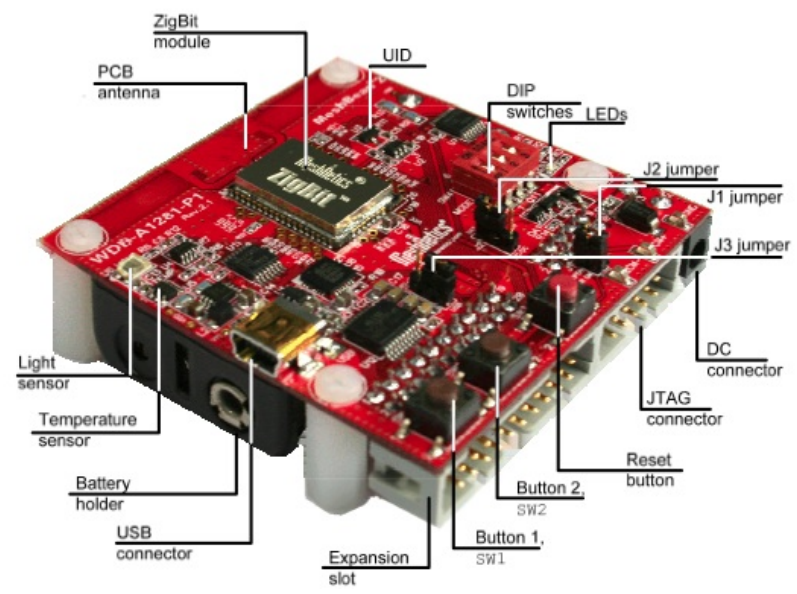

Fig. 7. Meshnetic mote scheme

Of all possible ZigBee topologies shown in previous section, the star ones is the most appropriated for our BSN design as shown in Fig. 4, being the mote connected to the personal server a $\mathrm{ZC}$ and the other motes ZED. 
At this point the sensors have acquired wireless connection capabilities. Next step is to provide them a common standardized interface. That is why the IEEE 1451 standard is used.

IEEE 1451.0 is mandatory in every system and as the communication among the sensor would be made through a wireless network it is needed to implement the 1451.5 .

IEEE 1451.0 supports the TEDS and services to acquire them by the Personal server. It includes the basic functions required to control and manage smart transducers, common communications protocols, and media-independent TEDS formats. It defines a set of implementation-independent APIs. As his functionality is independent of the physical communications media there is needed an standard involving that part. That is why it is needed the 1451.5, this standard provides the specification about the wireless communication methods in order to connect the 1451.0 implementations between the sensor and the personal server.

\section{Conclusion}

There has been presented a proposal for a telemedicine system. The BSN is designed to work with ZigBee because the low power consumption increase the autonomy of the different sensors. Even ZigBee has been used for real time systems in order to send data as humidity or temperature 3 large continuous data requires of good QOS policies for real time applications. There has been presented and described the main aspects of the IEEE 1451 standard which provides a common interface to acquire the data from the BSN. This standard makes easier the sensor network expansion using compliant sensors.

In order to provide wireless capabilities to wired sensors there has been used Meshnetic motes using BitCloud stack by Atmel. BitCloud provides the mechanisms to establish the network and deliver the information between the sensors and the personal server. At this scenarios the required implementation of the IEEE 1451standard concern to the sections IEEE 1451.0 and 1451.5 as it corresponds to the wireless connections.

\subsection{Acknowledgements}

This project has been carried out within the framework of two research programs: (p08-TIC-3631) Multimodal Wireless interface (IMI) funded by the Regional Government of Andalusia and Efficient and Health Intelligent Technologies Oriented to Health and comfort in Interior Environments (TECNO-CAI) approved project at the fifth call of CENIT program by the Innovation Science Ministry of Spain. 


\section{References}

1. D Ayyagari, Yongji $\mathrm{Fu}$, Jingping $\mathrm{Xu}$, and $\mathrm{N}$ Colquitt. Smart personal health manager: A sensor ban application: A demonstration. Consumer Communications and Networking Conference, 2009. CCNC 2009. 6th IEEE, pages 1-2, 2009.

2. C De Capua, A Meduri, and R Morello. A smart ecg measurement system based on web-service-oriented architecture for telemedicine applications. Instrumentation and Measurement, IEEE Transactions on, $\mathrm{PP}(99): 1$ - 9, 2010.

3. Jin Soo Choi and MengChu Zhou. Performance analysis of zigbee-based body sensor networks. Systems Man and Cybernetics (SMC), 2010 IEEE International Conference on

4. S Farshchi, A Pesterev, P Nuyujukian, I Mody, and J Judy. Bi-fi: An embedded sensor/system architecture for remote biological monitoring. Information Technology in Biomedicine, IEEE Transactions on, 11(6):611 - 618, 2007.

5. Fei Hu, S Lakdawala, Qi Hao, and Meikang Qiu;. Low-power, intelligent sensor hardware interface for medical data preprocessing. Information Technology in Biomedicine, IEEE Transactions on, 13(4):656 - 663, 2009.

6. Digital Imaging and communications in Medicine. http://medical.nema.org/.

7. Health Level Seven International. http://www.hl7.org/.

8. V Jeličić and V Bilas. Reducing power consumption of image transmission over ieee 802.15.4/zigbee sensor network. Instrumentation and Measurement Technology Conference (I2MTC), 2010 IEEE, pages 1211 - 1215, 2010.

9. Jiehui Jiang, Zhuangzhi Yan, Jun Shi, and P Kandachar. Design of wireless mobile monitoring of blood pressure for underserved in china by using short messaging service. Information Technology and Applications in Biomedicine, 2008. ITAB 2008. International Conference on, pages 319 - 322, 2008.

10. Yong-Zheng Li, Lei Wang, Xiao-Ming Wu, and Yuan-Ting Zhang;. Experimental analysis on radio transmission and localization of a zigbee-based wireless healthcare monitoring platform. Information Technology and Applications in Biomedicine, 2008. ITAB 2008. International Conference on, pages 488 - 490, 2008.

11. Messina. Implementing and validating an environmental and health monitoring system. Information Technology: New Generations, 2008. ITNG 2008. Fifth International Conference on, pages 994 - 999, 2008.

12. IEEE Standards Association page. http://standards.ieee.org/.

13. A Sapio and G Tsouri. Low-power body sensor network for wireless ecg based on relaying of creeping waves at $2.4 \mathrm{ghz}$. Body Sensor Networks (BSN), 2010 International Conference on, pages 167 - 173, 2010.

14. Dong Ik Shin, Soo Jin Huh, and Pil June Pak;. Patient monitoring system using sensor network based on the zigbee radio. Information Technology Applications in Biomedicine, 200\%. ITAB 200\%. 6th International Special Topic Conference on, pages $313-315,2007$.

15. NIST IEEE-P1451 Draft Standard. http://ieee1451.nist.gov/.

16. Xiaoxin Xu, Mingguang Wu, Cheng Ding, Bin Sun, and Jiangwei Zhang;. Outdoor wireless healthcare monitoring system for hospital patients based on zigbee. Industrial Electronics and Applications (ICIEA), 2010 the 5th IEEE Conference on, pages 549 - 554, 2010. tiene una minidescripción interesante de zigbee. 